\title{
Comparative Study of Two Short-Form Versions of the Montreal Cognitive Assessment for Screening of Post-Stroke Cognitive Impairment in a Chinese Population
}

This article was published in the following Dove Press journal: Clinical Interventions in Aging

\author{
Jingjing Wei ${ }^{1-3, *}$ \\ Xianglan Jin ${ }^{2, *}$ \\ Baoxin Chen ${ }^{2}$ \\ Xuemei $\mathrm{Liu}^{4}$ \\ Hong Zheng ${ }^{4}$ \\ Rongjuan Guo ${ }^{2}$ \\ Xiao Liang ${ }^{3}$ \\ Chen $\mathrm{Fu}^{2,4}$ \\ Yunling Zhang ${ }^{2,3}$ \\ 'Beijing University of Chinese Medicine, \\ Beijing, People's Republic of China; \\ ${ }^{2}$ Department of Neurology, Dongfang \\ Hospital, Beijing University of Chinese \\ Medicine, Beijing, People's Republic of \\ China; ${ }^{3}$ Department of Neurology, \\ Xiyuan Hospital, China Academy of \\ Chinese Medical Sciences, Beijing, \\ People's Republic of China; ${ }^{4}$ Central \\ Laboratory, Dongfang Hospital, Beijing \\ University of Chinese Medicine, Beijing, \\ People's Republic of China
}

*These authors contributed equally to this work

Correspondence: Yunling Zhang Department of Neurology, Xiyuan Hospital, China Academy of Chinese Medical Sciences, I Xiyuan Playground, Haidian District, Beijing 10009I, People's

Republic of China

Tel/Fax +86-10-6283-5960

Email yunlingzhang2004@I26.com

Chen Fu

Central Laboratory, Dongfang Hospital, Beijing University of Chinese Medicine, 6 First Block, Fangxingyuan, Fengtai District, Beijing 100078, People's Republic of China

Tel +86-10-6768-9634

Fax +86-10-67691949

Email fuchen2003@I63.com
Purpose: Cognitive impairment (CI) is one of the most significant post-stroke complications. The Montreal Cognitive Assessment (MoCA) is widely applied to the early screening of post-stroke CI (PSCI), and has good sensitivity and specificity, but needs a long time to administer. Clinicians and researchers need shorter, more effective cognitive testing tools. The purpose of this study was to detect the sensitivity and specificity of two different shortform versions of the MoCA (SF-MoCA) for screening of PSCI in a Chinese population.

Methods: A total of 2,989 stroke participants were included from 14 hospitals in northern and southern China between June 2011 and September 2013. The sensitivity and specificity of the two SF-MoCA versions were compared.

Results: Using an MoCA score $<26$ as the critical value, the National Institute of Neurological Disease and Stroke-Canadian Stroke Network SF-MoCA showed sensitivity of $91 \%$ and specificity of $63 \%$ (PPV $71 \%$, BPV $87 \%$ ) with scores $\leq 10$ points. The sensitivity and specificity of the Bocti SF-MoCA were $92 \%$ and $69 \%$ (PPV 75\%, BPV 89\%) with scores $\leq 7$, respectively. The area under the curve was 0.885 (95\% CI $0.873-0.897)$ and $0.912(95 \%$ CI 0.902-0.922), respectively.

Conclusion: The Bocti SF-MoCA can be used as a briefer and more effective screening tool for PSCI in Chinese.

Keywords: cognitive dysfunction, Montreal Cognitive Assessment, MoCA, stroke, sensitivity, specificity

\section{Plain-Language Summary}

The Montreal Cognitive Assessment (MoCA) is widely applied to the early screening of post-stroke cognitive impairment (PSCI), but it is time-consuming and not routinely carried out. Clinicians and researchers need shorter, more effective cognitive testing tools. National Institute of Neurological Disease and Stroke-Canadian Stroke Network once proposed a 5-minute protocol, which has been verified as brief, valid, reliable, and feasible for telephone administration in detecting PSCI. The Bocti SF-MoCA has also demonstrated reliability in detecting PSCI. However, there have been few published studies reporting the sensitivity and specificity of the two SF-MoCAs in the Chinese post-stroke population. In this study, we included 2,989 Chinese stroke patients and compared the sensitivity and specificity of the two SF-MoCAs. Our results showed that both SF-MoCAs had good reliability. Interestingly, Bocti SF-MoCA was better on both sensitivity and specificity, 
which is worth further research, and we conclude that the Bocti SF-MoCA could be used as a briefer and more effective screening tool for PSCI in Chinese.

\section{Introduction}

Cognitive impairment (CI), one of the most significant poststroke complications, occurs at any time after stroke, even after incident stroke, ${ }^{1}$ up to 10 years of follow-up. ${ }^{2}$ A third of stroke survivors may be troubled by post-stroke CI (PSCI) or post-stroke dementia. ${ }^{3}$ Evidence has indicated that first-ever mild stroke might affect cognition and daily functioning, consequently affecting participation, quality of life, and return to work, ${ }^{4}$ and incidence of dementia being 50 times that in the year after a major stroke compared with the general population. ${ }^{5}$ Although there is little evidence for specific therapeutic strategies for preventing cognitive decline after stroke, researches have shown that starting a rehabilitation course for $\mathrm{CI}$ as early as possible has a pivotal role in the outcome of patients. ${ }^{6-8}$ Therefore, early screening and recognition of PSCI is very important for patients' rehabilitation treatment.

To date, the Mini-Mental State Examination (MMSE) and Montreal Cognitive Assessment (MoCA) have been the most reasonable instruments for identifying PSCI. ${ }^{9}$ Researches has shown that the MoCA has better sensitivity in detecting PSCI,${ }^{10-15}$ especially in the Chinese population. ${ }^{16,17}$ Our preliminary study ${ }^{18}$ proved that the Beijing version of the MoCA executive tasks were more sensitive in detecting post-stroke executive dysfunction compared with MMSE executive tasks. In all, the MoCA is the most widely used and reliable scale in identifying PSCI.

An important concern is that completion of the MoCA can take much longer in practice ${ }^{19}$ than 10 minutes. A shorter form of the MoCA (SF-MoCA) is attractive for both patients and clinicians, especially in China, considering stroke burden has increased over the last 30 years in China and the country bears the biggest stroke burden in the world (1,596 per 100,000 people). ${ }^{20}$ Therefore, many studies have been devoted to SF-MoCAs, since they could improve clinical feasibility, shorten diagnosis time, and reduce assessment burden on patients. A recent study ${ }^{21}$ evaluated the comparative performance of seven SF-MoCAs in identifying mild CI or dementia, and the results showed that all seven had acceptable performance in participants aged $\geq 50$ years. Another cross-sectional test study proposed a brief four-item MoCA that could be a good alternative in nonspecialty clinics. ${ }^{22}$ Moreover, a recent systematic review ${ }^{23}$ containing 13 SF-
MoCAs suggested that the choice of SF-MoCAs should be varied by clinical population.

All in all, it is very important to conduct comparative studies of SF-MoCAs in different populations to choose the optimal SF-MoCA. As early as in 2006, the National Institute of Neurological Disease and Stroke-Canadian Stroke Network VCI Harmonization Working Group proposed a 5-minute protocol (NINDS-CSN SF-MoCA) ${ }^{24}$ which was subsequently verified as brief, valid, reliable, and feasible for telephone administration in detecting PSCI. ${ }^{25}$ The Bocti SFMoCA has also demonstrated reliability in detecting PSCI. ${ }^{26,27}$ However, there have been few published studies reporting on the sensitivity and specificity of the two SFMoCAs in the Chinese post-stroke population. Our study focused on the sensitivity, specificity, and comparison of the two SF-MoCAs by a cross-sectional large-sample screening in a Chinese population. The purpose of this study was to provide shorter and more effective time-saving screening tools in detecting PSCI for clinicians.

\section{Methods}

\section{Study Design and Participants}

Data were obtained from the "Study on Traditional Chinese Medicine (TCM) prevention, treatment and administration in community of post-stroke cognitive impairment" project, which is supported by the TCM Special Research Projects Program, China Ministry of Science and Technology (201007002). As our pervious published study, ${ }^{18}$ patients (2 weeks to 6 months after stroke) were recruited from 14 hospitals in northern and southern China (ten top-grade hospitals and four community hospitals) between June 2011 and September 2013, and their cognitive status was scored with the Beijing version of the MoCA.

PSCI emphasizes the importance of stroke as a vascular factor leading to CI. The diagnosis of PSCI was conducted by neurologists using the NINDS-CSN VCI standard ${ }^{24}$ on the basis of neuropsychological examination together with other available clinical information, such as medical history, prestroke cognitive functioning, and cognition-related functional decline. A MoCA score $<26$ was taken to indicate CI in stroke patients according to recommendations from published data. ${ }^{14,28}$ Also, another stroke cross-sectional study in China has cited this standard. ${ }^{29}$ The two SF-MoCA scores were derived retrospectively from the full MoCA.

Inclusion criteria were complaining of $\mathrm{CI}$ or $\mathrm{CI}$ reported by caregiver(s), conscious without communication problems hindering the performance of cognitive tests (eg, aphasia, 
apraxia, visual deprivation, hearing loss), and cooperative during neuropsychological examination. Exclusion criteria were Hamilton Depression Scale score $\geq 17$ point, acute mental changes (eg, delirium), CI before stroke recorded in medical documents or reported by patients and families, history of head trauma, brain tumor, epilepsy, schizophrenia, or other psychiatric disorders, drug abuse, substance abuse, or alcohol addiction, and moderate-severe dementia. All participants signed informed consent. The study was approved by the Ethics Committee on Clinical Research of Dongfang Hospital (2011123004).

\section{Procedure}

General data on all subjects — sex, birth date, education, history of smoking or alcohol intake, as well as history of hypertension, diabetes mellitus, coronary heart disease, previous stroke/transient ischemic attack, and peripheral vascular diseases — were obtained. All staff designated for assessment underwent strict professional training courses. Participants were instructed to complete the Beijing version of the MoCA and received clinical and functional assessment, which took approximately 30 minutes. Each participant was instructed to complete the MoCA first, and after at least an hour they were instructed to complete the MMSE. ${ }^{24}$

The Beijing version of the MoCA ${ }^{30,31}$ (http://www.mocat est.org) contains seven cognitive domains (visuospatial/ executive function, naming, attention, abstraction, language, delayed recall, and orientation) translated from the original English version literally, with the exception of the following modifications. For the visuospatial/executive function domain, alphabetic letters are replaced by Chinese characters (甲/乙/丙/丁/戊), which denote the same as “A/B/C/D/E” in English. For the attention domain, numbers are used instead of alphabetic letters in the auditory vigilance task. For the language domain, in the verbal fluency task, the phonemic fluency task that requires participants to generate words beginning with the letter $\mathrm{F}$ is replaced by the semantic fluency task, requiring participants to produce as many animals as possible in 60 seconds. When education of the participants was $<12$ years (high middle school), their MoCA score would have 1 point added. Activities of daily living (ADL) including basic ADL and instrumental ADL, National Institute of Health Stroke Scale (NIHSS), and Hamilton Depression Scale were also assessed.

\section{Statistical Analysis}

All statistical analysis was performed using SPSS 20.0 software (IBM, Armonk, NY, USA). Continuous and categorical variables are presented as means \pm SD and frequency (\%), respectively. Nonparametric and $\chi^{2}$ tests were used to compare two groups for continuous and categorical variables, respectively.The diagnostic accuracy of the two SF-MoCAs for prediction of clinical diagnosis of PSCI was assessed through receiver- operating characteristic (ROC)-curve analysis, with larger area under the curve (AUC) indicating better diagnostic accuracy. ROC curves were compared according to the AUC-comparison method of Hanley and McNeil. ${ }^{32}$ Optimal cutoff points for each screening instrument that yielded the highest Youden index values were selected, with higher values indicating maximum sensitivity and specificity. To analyze the predictive value of the tests, for each cutoff point we calculated the sensitivity (probability of subjects with CI having a positive result), specificity (probability of subjects without CI having a negative test), positive predictive value (PPV; probability of disease in subjects with a positive result), negative predictive value (NPV; probability of a "lack of disease" classification in subjects with a negative result), and classification accuracy (probability of correct classification of subjects, with or without CI). ${ }^{33}$ All $P$-values were considered statistically significant if $P<0.05$.

\section{Results}

A total of 3,000 participants were recruited for this study, of which eleven were excluded due to incomplete data (six did not complete the MoCA, five lacking imaging data), and so 2,989 were eventually included for statistical analysis. There were 1,651 males of mean age $62.50 \pm 10.56$ years (range 33-92 years) and 1,338 females of mean age $63.56 \pm 9.92$ years (range 33-90 years). Median stroke duration was $1-2$ months. Mean NIHSS score was 1.16 \pm 2.54 , ADL 25.67 \pm 11.23 , Hamilton Depression Scale $3.68 \pm 3.88$, and MMSE 27.24 \pm 2.83 . The range of MMSE scores was 17-30 (Table 1). These patients had different lesion regions, lesion sides, lesion size, and types of stroke, as shown in Table 2. The severity of these patients was examined by the NIHSS and ADL scale, which were $1.16 \pm 2.54$ and $25.67 \pm 11.23$ respectively. Vascular risk factors are shown in Table 3.

Table 4 shows descriptive statistics for each test of cognition, including the MMSE, MoCA total, and every MoCA subset. After division of patients into two groups by cutoff score for the MoCA of 26, the prevalence of PSCI was 50.4\% (1,506 of 2,989), and all cognitive domains in the MoCA score $<26$ group (visuospatial/executive, naming, attention, language, abstraction, delayed recall, and orientation) scores 
Table I Demographic Characteristics and Test Scores

\begin{tabular}{|l|l|}
\hline & Overall (n=2,989) \\
\hline Age (years) & $63.03 \pm 10.28(33-92)$ \\
\hline Sex & \\
$\quad$ Male (\%) & $1,651(55.2)$ \\
Female (\%) & $1,338(44.8)$ \\
\hline Education (\%) & \\
$<6$ years & $716(24.0)$ \\
6-I2 years & $826(27.6)$ \\
$\geq 12$ years & $1,447(48.4)$ \\
\hline BMI (kg/m $\left.{ }^{2}\right)$ & $24.28 \pm 3.01$ \\
NIHSS & $1.16 \pm 2.54$ \\
ADL scale & $25.67 \pm 11.23$ \\
HAMD & $3.68 \pm 3.88$ \\
MMSE & $27.24 \pm 2.83(17-30)$ \\
\hline
\end{tabular}

Abbreviations: BMI, Body Mass Index; NIHSS, National Institutes of Health Stroke Scale; ADL, Activities of Daily Living; HAMD, Hamilton Depression (17 items); MMSE, Mini-Mental State Examination.

Table 2 Lesion Regions, Lesion Sides, and Types of Stroke

\begin{tabular}{|l|l|}
\hline & Overall (n=2,989) \\
\hline $\begin{array}{l}\text { Lesions involving structure } \\
\text { Cortex }\end{array}$ & $333(11.1 \%)$ \\
Subcortical white matter & $800(26.8 \%)$ \\
Basal ganglia & $1,796(60.1 \%)$ \\
Thalamus & $204(6.8 \%)$ \\
Brain stem & $215(7.2 \%)$ \\
Cerebellum & $99(3.3 \%)$ \\
\hline Lesions involving lobe & \\
Frontal & $570(19.1 \%)$ \\
Parietal & $507(17.0 \%)$ \\
Temporal & $350(11.7 \%)$ \\
Occipital & $153(5.1 \%)$ \\
Insular & $42(1.4 \%)$ \\
\hline Lesion side & \\
Left & $679(22.7 \%)$ \\
Right & $667(22.3 \%)$ \\
Bilateral & $1,456(48.7 \%)$ \\
\hline Type of stroke & \\
Ischemic & $2,867(95.9 \%)$ \\
Hemorrhagic & $122(4.1 \%)$ \\
\hline
\end{tabular}

were lower than MoCA score $\geq 26$ group. As shown in Table 5, when the NINDS-CSN SF-MoCA score was 10/12 or less, sensitivity was $91 \%$ and specificity $63 \%$ (PPV $71 \%$, NPV $87 \%$ ), and when the Bocti SF-MoCA score was $7 / 10$ or less, sensitivity was $92 \%$ and specificity was $69 \%$ (PPV $75 \%$, NPV 89\%). The AUC was 0.885 (95\% CI 0.873-0.897) and 0.912
Table 3 Vascular Risk Factors

\begin{tabular}{|l|l|l|}
\hline & Yes & No \\
\hline Smoking & $1,043(34.9 \%)$ & $1,946(65.1 \%)$ \\
Alcohol & $961(32.2 \%)$ & $2,028(67.8 \%)$ \\
Hypertension & $1,989(66.5 \%)$ & $1,000(33.5 \%)$ \\
Diabetes & $695(23.3 \%)$ & $2,294(76.7 \%)$ \\
Dyslipidemia & $691(23.1 \%)$ & $2,298(76.9 \%)$ \\
Heart diseases & $535(17.9 \%)$ & $2,454(82.1 \%)$ \\
Previous stroke/TIA & $545(18.2 \%)$ & $2,444(71.8 \%)$ \\
Peripheral vascular diseases & $37(1.2 \%)$ & $2,952(98.8 \%)$ \\
\hline
\end{tabular}

Abbreviation: TIA, transient ischemic attack.

(95\% CI 0.902-0.922), respectively. Table 6 is a crossclassification of patients by each SF-MoCA.

\section{Discussion}

To our knowledge, this is the first study to explore the utility and accuracy of these two SF-MoCAs in a Chinese poststroke population. The main findings of our study were that sensitiviy and specificity of the NINDS-CSN SF-MoCA were $91 \%$ and $63 \%$ and AUC 0.885 (95\% CI 0.873-0.897), sensitivity and specificity of the Bocti SF-MoCA were $92 \%$ and $69 \%$ and AUC 0.912 (95\% CI 0.902-0.922), both the SF-MoCAs had high sensitivity and low specificity, and the Bocti SF-MoCA was superior in detecting PSCI in this Chinese population.

\section{Sensitivity and Specificity of NINDS-CSN SF-MoCA}

The NINDS-CSN SF-MoCA, known as the NINDS-CSN 5-minute protocol, consists of only verbally conducted tests: orientation, memory, and verbal fluency tasks. As such, it has been recommended for stroke patients for whom paper-and-pencil tests were inconvenient or who needed telephone follow-up. ${ }^{24,25}$ In our study, a cutoff of ten scores was identified as optimal (sensitivity $91 \%$, specificity $63 \%$, AUC 0.885 ) based on ROC-curve analysis, which was different from a previous study ${ }^{34}$ that applied the NINDS-CSN 5-minute neuropsychology protocol in acute stroke for detecting post-stroke dementia, with an acceptable value was seven scores. Possible reasons for the difference may be that our subjects were at different stages of stroke (2 weeks to 6 months vs within 2 weeks) and the coverage of disease (PSCI vs post-stroke dementia) was different. Also, this difference reminds us that the sensitivity and specificity of the NINDS-CSN SF-MoCA may vary according to the clinical population and research purposes, 
Table 4 Descriptive Statistics for Each Test Factor of the MoCA

\begin{tabular}{|c|c|c|c|c|c|}
\hline Domain & $\begin{array}{l}\text { All Patients, } \\
\mathrm{n}=\mathbf{2 , 9 8 9}\end{array}$ & $\begin{array}{l}\text { MoCA <26, } \\
n=1,506\end{array}$ & $\begin{array}{l}M o C A \geq 26, \\
n=1,483\end{array}$ & $z$ & $P$ \\
\hline \multicolumn{6}{|c|}{ Visuospatial/executive (VE) } \\
\hline Trail-making & $0.47 \pm 0.50$ & $0.28 \pm 0.45$ & $0.67 \pm 0.47$ & 21.096 & $0 *$ \\
\hline Cube copy & $0.63 \pm 0.48$ & $0.46 \pm 0.50$ & $0.79 \pm 0.4 I$ & 18.792 & $0^{*}$ \\
\hline Clock-drawing & $2.45 \pm 0.84$ & $2.10 \pm 0.97$ & $2.80 \pm 0.46$ & 23.152 & $0 *$ \\
\hline Subtotal (VE) & $3.55 \pm 1.36$ & $2.84 \pm 1.40$ & $4.26 \pm 0.85$ & 28.680 & $0 *$ \\
\hline Naming & $2.76 \pm 0.52$ & $2.59 \pm 0.65$ & $2.93 \pm 0.25$ & 18.269 & $0 *$ \\
\hline \multicolumn{6}{|l|}{ Attention } \\
\hline Digit span & $\mathrm{I} .84 \pm 0.4 \mathrm{I}$ & $I .7 I \pm 0.52$ & $1.98 \pm 0.14$ & 19.325 & $0^{*}$ \\
\hline Letter $\mathrm{A}$ & $0.83 \pm 0.38$ & $0.74 \pm 0.44$ & $0.92 \pm 0.28$ & 12.797 & $0^{*}$ \\
\hline Serial 7 & $2.60 \pm 0.72$ & $2.32 \pm 0.85$ & $2.89 \pm 0.38$ & 23.439 & $0^{*}$ \\
\hline Subtotal (attention) & $5.27 \pm 1.11$ & $4.76 \pm 1.30$ & $5.79 \pm 0.48$ & 26.494 & $0 *$ \\
\hline \multicolumn{6}{|l|}{ Language } \\
\hline Sentence repeat & $|.46 \pm 0.7|$ & $1.15 \pm 0.76$ & $1.76 \pm 0.48$ & 23.818 & $0 *$ \\
\hline Fluency & $0.85 \pm 0.36$ & $0.76 \pm 0.43$ & $0.94 \pm 0.24$ & 13.746 & $0^{*}$ \\
\hline Subtotal (language) & $2.30 \pm 0.83$ & $1.91 \pm 0.88$ & $2.70 \pm 0.52$ & 26.589 & $0^{*}$ \\
\hline Abstraction & $1.44 \pm 0.68$ & $1.16 \pm 0.73$ & $\mathrm{I} .73 \pm 0.47$ & 22.834 & $0 *$ \\
\hline Delayed recall & $3.04 \pm 1.44$ & $2.19 \pm 1.38$ & $3.91 \pm 0.86$ & 33.417 & $0 *$ \\
\hline Orientation & $5.62 \pm 0.88$ & $5.34 \pm 1.13$ & $5.91 \pm 0.32$ & 19.619 & $0 *$ \\
\hline MoCA total & $24.26 \pm 4.27$ & $21.10 \pm 3.83$ & $27.46 \pm 1.18$ & 47.569 & $0 *$ \\
\hline
\end{tabular}

Note: Data are means \pm SD, $* P<0.05$.

Abbreviation: MoCA, Montreal Cognitive Assessment.

which was similar to the results of existing researches. $^{27,33,35,36}$

\section{Sensitivity and Specificity of Bocti SF-MoCA}

The Bocti SF-MoCA includes verbal fluency, cube copy, trail-making, abstraction, and five-word recall. A previous study $^{26}$ took Canadian stroke patients after 3 months as

Table 5 Sensitivity, Specificity, and Positive and Negative Predictive Values of the Two SF-MoCAs

\begin{tabular}{|l|l|l|l|l|}
\hline & Sensitivity & Specificity & PPV & NPV \\
\hline NINDS-CSN SF-MoCA & $91 \%$ & $63 \%$ & $71 \%$ & $87 \%$ \\
$\leq 10 / 12$ & & & & \\
NINDS-CSN SF-MoCA $\leq 9 / 12$ & $71 \%$ & $93 \%$ & $91 \%$ & $76 \%$ \\
NINDS-CSN SF-MoCA $\leq 8 / 12$ & $46 \%$ & $99 \%$ & $97 \%$ & $64 \%$ \\
Bocti SF-MoCA $\leq 8 / 10$ & $98 \%$ & $34 \%$ & $60 \%$ & $93 \%$ \\
Bocti SF-MoCA $\leq 7 / 10$ & $92 \%$ & $69 \%$ & $75 \%$ & $89 \%$ \\
Bocti SF-MoCA $\leq 6 / 10$ & $77 \%$ & $90 \%$ & $89 \%$ & $80 \%$ \\
\hline
\end{tabular}

Notes: NINDS-CSN SF-MoCA score (12 points) includes orientation (6 points), five-word recall (5 points), and word fluency (I point); Bocti SF-MoCA score (10 points) includes verbal fluency (I point), cube copy (I point), trail-making (I point), abstraction (2 points), and five-word recall (5 points).

Abbreviations: NINDS-CSN, National Institute of Neurological Disorders and Stroke-Canadian Stroke Network; MoCA, Montreal Cognitive Assessment; SFMoCA, short-form MoCA. subjects and sensitivity was $91 \%$, similar to our result. Considering the median stroke duration in our study was 1-2 months, this is not surprising. Also, there is no "best" value. For high sensitivity, we choose eight scores as the cutoff so as not to miss patients who needed further indepth assessment. All in all, the Bocti SF-MoCA is also suitable for detecting PSCI in Chinese.

\section{Comparison of NINDS-CSN SF-MoCA and Bocti SF-MoCA}

SF-MoCAs provide a viable alternative for detection of PSCI when it is not feasible to complete the full MoCA in clinical practice. SF-MoCAs are especially useful in nonspecialty clinics (such as primary-care clinics) ${ }^{21}$ for early detection of $\mathrm{CI}$, since they are derived from the full MoCA, time-saving, and less resource-consuming. Screen positives mean further in-depth professional neuropsychological evaluations are needed, and also provide the possibility of timely and early interventions, which is in line with the International Association of Gerontology and Geriatrics call ${ }^{37}$ on the need for validated short screening tests and timely support for early CI. As shown in Table 5, both the Bocti SF-MoCA and NINDS-CSN SFMoCA had lower specificity and higher sensitivity, with 
Table 6 Cross Classification of Patients Screening Positive or Negative for Cognitive Impairment on the Two SF-MoCAs

\begin{tabular}{|c|l|l|}
\hline \multirow{2}{*}{} & \multicolumn{2}{|l|}{ MoCA Score } \\
\cline { 2 - 3 } & $<26$ & $\geq 26$ \\
\hline $\begin{array}{c}\text { NINDS-CSN SF-MoCA } \\
\leq 10\end{array}$ & $1,363(45.5 \%)$ & $556(18.6 \%)$ \\
$>10$ & $143(4.8 \%)$ & $927(31.0 \%)$ \\
\hline $\begin{array}{c}\text { Bocti SF-MoCA } \\
\leq 7\end{array}$ & $1,381(46.2 \%)$ & $467(15.6 \%)$ \\
$>7$ & $125(4.2 \%)$ & $1,016(34.0 \%)$ \\
\hline
\end{tabular}

Abbreviations: NINDS-CSN, National Institute of Neurological Disorders and Stroke-Canadian Stroke Network; MoCA, Montreal Cognitive Assessment; SFMoCA, short-form MoCA.

corresponding low positive predictive value and high negative predictive value. This was consistent with a newly published systematic review. ${ }^{23}$ These results were not surprising, since the MoCA was designed to detect mild CI and our participants included people with mild dementia. High sensitivity suggests potential utility of the two SF-MoCAs as initial cognitive screening tools, but low specificity suggests many false positives and further assessment is necessary.

Compared with the NINDS-CSN MoCA, the Bocti SFMoCA includes executive function (ie, cube copy, trailmaking) besides memory and word fluency. Therefore, the Bocti SF-MoCA picked up substantially more cognitive deficits in patients with stroke than the NINDS-CSN MoCA. Moreover, in the years following an incident stroke, executive function declines significantly faster than it did before the stroke, ${ }^{1}$ and $40.6 \%$ of post-ischemic stroke patients had executive dysfunction. ${ }^{38}$ That is to say, executive dysfunction has become one of the most significant PSCI complications, ${ }^{24,39,40}$ and the detection of executive function is of great significance after stroke. In the present study, we found that the sensitivity and specificity of the Bocti SF-MoCA were higher than the NINDS-CSN SF-MoCA. From Table 6, we can also see that the Bocti SF-MoCA had greater true-positive and -negative rates. Te AUC of the Bocti SF-MoCA was also more accurate. These findings showed that including more cognitive domains, especially executive, may be one advantage of the Bocti SFMoCA.

\section{Limitations}

In this study, several limitations existed. Firstly, we used just MoCA scores $<26$ as a cutoff for diagnosis, and did not administer an independent and more extensive neuropsychological battery as a gold standard. Therefore, we cannot be certain that lower scores on the MoCA were detecting meaningful CI. This might bring a risk of circularity. More accurate assessments of PSCI should be added in future studies (such as the 60-minute protocol). ${ }^{24}$ Secondly, NIHSS scores of subjects in this study were $1.16 \pm 2.54$ on average, and may not be applicable to patients with severe stroke, who may be more concerned about survival rate. ${ }^{41}$ Thirdly, patients with moderate and severe dementia were excluded from the present study, because of the ceiling effect. Finally, the choice of cutoff points should depend on whether the test is used as a screen (high sensitivity required) or as a diagnostic tool (high specificity required). ${ }^{42}$ Also, we conducted this cross-sectional study on only two SF-MoCAs, both of which were derived retrospectively from the full MoCA.Further investigations with prospective design are needed to clarify more reliable cutoff points for SF-MoCAs according to different clinical environments.

\section{Conclusion}

The Bocti SF-MoCA - trail-making (1 point), cube copy (1 point), verbal fluency (1 point), abstraction ( 2 points), and five-word recall ( 5 points) — can be used as a briefer and more effective screening tool for Chinese PSCI patients.

\section{Acknowledgments}

This study was funded by National Adiministration of TCM (NATCM) TCM Inheritance and Innovation "HundredThousuand-Ten Thousand" Talents Project (Qihuang Scholar)-National TCM Leading Personnel Support Program (NATCM Personnel and Education Department[2018]12), the TCM Special Research Projects Program, State Administration of TCM (201007002, 201407001-8), and National Key R\&D Program of China (2018YFC1704300). We are also grateful to the participants in this study for their generous participation and their clinicians for recruiting them in this study.

\section{Disclosure}

The authors report no conflicts of interest in this work.

\section{References}

1. Levine DA, Galecki AT, Langa KM, et al. Trajectory of cognitive decline after incident stroke. JAMA. 2015;314(1):41. doi:10.1001/ jama.2015.6968

2. Ivan CS, Seshadri S, Beiser A, et al. Dementia After Stroke. Stroke. 2004;35(6):1264-1268. doi:10.1161/01.STR.0000127810.92616.78

3. Gorelick PB, Nyenhuis D. Stroke and cognitive decline. JAMA. 2015;314(1):29-30. doi:10.1001/jama.2015.7149 
4. Fride Y, Adamit T, Maeir A, et al. What are the correlates of cognition and participation to return to work after first ever mild stroke? Top Stroke Rehabil. 2015;22(5):317-325. doi:10.1179/107493 5714Z.0000000013

5. Pendlebury ST, Rothwell PM, Oxford VS. Incidence and prevalence of dementia associated with transient ischaemic attack and stroke: analysis of the population-based oxford vascular study. Lancet Neurol. 2019;18(3):248-258. doi:10.1016/S1474-4422(18)30442-3

6. Boss HM, Van Schaik SM, Deijle IA, et al. A randomised controlled trial of aerobic exercise after transient ischaemic attack or minor stroke to prevent cognitive decline: the MoveIT study protocol. BMJ Open. 2014;4(12):e7065. doi:10.1136/bmjopen-2014-007065

7. Muresanu DF, Heiss W, Hoemberg V, et al. Cerebrolysin and Recovery After Stroke (CARS). Stroke. 2016;47(1):151-159. doi:10.1161/STROKEAHA.115.009416

8. Paolucci S, Antonucci G, Grasso MG, et al. Early versus delayed inpatient stroke rehabilitation: a matched comparison conducted in Italy. Arch Phys Med Rehabil. 2000;81(6):695-700. doi:10.1016/ S0003-9993(00)90095-9

9. Cumming TB, Churilov L, Linden T, Bernhardt J. Montreal cognitive assessment and mini-mental state examination are both valid cognitive tools in stroke. Acta Neurol Scand. 2013;128(2):122-129. doi:10.1111/ane.12084

10. Stolwyk RJ, O Neill MH, McKay AJD, Wong DK. Are cognitive screening tools sensitive and specific enough for use after stroke? Stroke. 2014;45(10):3129-3134. doi:10.1161/STROKEAHA.1 14.004232

11. Salvadori E, Pasi M, Poggesi A, Chiti G, Inzitari D, Pantoni L. Predictive value of MoCA in the acute phase of stroke on the diagnosis of mid-term cognitive impairment. J Neurol. 2013;260 (9):2220-2227. doi:10.1007/s00415-013-6962-7

12. Lees R, Selvarajah J, Fenton C, et al. Test accuracy of cognitive screening tests for diagnosis of dementia and multidomain cognitive impairment in stroke. Stroke. 2014;45(10):3008-3018. doi:10.1161/ STROKEAHA.114.005842

13. Jaywant A, Toglia J, Gunning FM, O’Dell MW. The diagnostic accuracy of the Montreal Cognitive Assessment in inpatient stroke rehabilitation. Neuropsychol Rehabil. 2019;29(8):1163-1176. doi:10.1080/09602011.2017.1372297

14. Pendlebury ST, Cuthbertson FC, Welch SJV, Mehta Z, Rothwell PM. Underestimation of cognitive impairment by mini-mental state examination versus the montreal cognitive assessment in patients with transient ischemic attack and stroke. Stroke. 2010;41(6):1290-1293. doi:10.1161/STROKEAHA.110.579888

15. Chiti G, Pantoni L. Use of montreal cognitive assessment in patients with stroke. Stroke. 2014;45(10):3135-3140. doi:10.1161/STROKEAHA.114. 004590

16. Zhao J, Tang H, Sun J, Wang B, Chen S, Fu Y. Analysis of cognitive dysfunction with silent cerebral infarction: a prospective study in Chinese patients. Metab Brain Dis. 2012;27(1):17-22. doi:10.1007/ s11011-011-9275-5

17. Tu Q, Jin H, Ding B, et al. Reliability, validity, and optimal cutoff score of the montreal cognitive assessment (Changsha version) in ischemic cerebrovascular disease patients of Hunan Province, China. Dement Geriatr Cogn Dis Extra. 2013;3(1):25-36. doi:10.1159/ 000346845

18. Fu C, Jin X, Chen B, et al. Comparison of the Mini-Mental State Examination and Montreal Cognitive Assessment executive subtests in detecting post-stroke cognitive impairment. Geriatr Gerontol Int. 2017;17(12):2329-2335. doi:10.1111/ggi.13069

19. Blackburn DJ, Bafadhel L, Randall M, Harkness KA. Cognitive screening in the acute stroke setting. Age Ageing. 2012;42 (1):113-116. doi:10.1093/ageing/afs116

20. Wang W, Jiang B, Sun H, et al. Prevalence, incidence, and mortality of stroke in China. Circulation. 2017;135(8):759-771. doi:10.1161/ CIRCULATIONAHA.116.025250
21. Liew TM. The optimal short version of montreal cognitive assessment in diagnosing mild cognitive impairment and dementia. J Am Med Dir Assoc. 2019;20(8):1051-1055. doi:10.1016/j.jamda.2019.02.004

22. Liew TM. A 4-item case-finding tool to detect dementia in older persons. J Am Med Dir Assoc. 2019;20(12):1529-1534. doi:10.1016/ j.jamda.2019.06.015

23. McDicken JA, Elliott E, Blayney G, et al. Accuracy of the short-form montreal cognitive assessment: systematic review and validation. Int J Geriatr Psych. 2019;34(10):1515-1525. doi:10.1002/gps.5162

24. Hachinski V, Iadecola C, Petersen RC, et al. National institute of neurological disorders and stroke-canadian stroke network vascular cognitive impairment harmonization standards. Stroke. 2006;37 (9):2220-2241. doi:10.1161/01.STR.0000237236.88823.47

25. Wong A, Nyenhuis D, Black SE, et al. Montreal cognitive assessment 5-minute protocol is a brief, valid, reliable, and feasible cognitive screen for telephone administration. Stroke. 2015;46(4):1059-1064. doi:10.1161/STROKEAHA.114.007253

26. Bocti C, Legault V, Leblanc N, et al. Vascular cognitive impairment: most useful subtests of the montreal cognitive assessment in minor stroke and transient ischemic attack. Dement Geriatr Cogn. 2013;36 (3-4):154-162. doi:10.1159/000351674

27. Campbell N, Rice D, Friedman L, Speechley M, Teasell RW. Screening and facilitating further assessment for cognitive impairment after stroke: application of a shortened Montreal Cognitive Assessment (miniMoCA). Disabil Rehabil. 2016;38(6):601-604. doi:10.3109/09638288.2015.1047968

28. Nasreddine ZS, Phillips NA, Bédirian V, et al. The Montreal Cognitive Assessment, MoCA: a brief screening tool for mild cognitive impairment. $J$ Am Geriatr Soc. 2005;53(4):695-699. doi:10.1111/j.1532-5415.2005.53221.x

29. Qu Y, Zhuo L, Li N, et al. Prevalence of post-stroke cognitive impairment in china: a community-based, cross-sectional study. PLoS One. 2015;10(4):e122864. doi:10.1371/journal.pone.0122864

30. Huang Y, Qian S, Guan Q, et al. Comparative study of two chinese versions of montreal cognitive assessment for screening of mild cognitive impairment. Appl Neuropsychol Adult. 2019;1-6.

31. Yu J, Li J, Huang X. The Beijing version of the Montreal Cognitive Assessment as a brief screening tool for mild cognitive impairment: a community-based study. BMC Psychiatry. 2012;12(1):156. doi:10.1186/1471-244X-12-156

32. Hanley JA, McNeil BJ. A method of comparing the areas under receiver operating characteristic curves derived from the same cases. Radiology. 1983;148(3):839-843. doi:10.1148/radiology.148. 3.6878708

33. Freitas S, Simões MR, Alves L, Vicente M, Santana I. Montreal Cognitive Assessment (MoCA): validation study for vascular dementia. J Int Neuropsych Soc. 2012;18(6):1031-1040. doi:10.101 7/S135561771200077X

34. Lim JS, Oh MS, Lee JH, et al. Prediction of post-stroke dementia using NINDS-CSN 5-minute neuropsychology protocol in acute stroke. Int Psychogeriatr. 2017;29(5):777-784. doi:10.1017/S10416 10216002520

35. Cameron JD, Gallagher R, Pressler SJ, et al. Sensitivity and specificity of a five-minute cognitive screening test in patients with heart failure. J Card Fail. 2016;22(2):99-107. doi:10.1016/j.cardfail. 2015.08.343

36. Pendlebury ST, Welch SJ, Cuthbertson FC, Mariz J, Mehta Z, Rothwell PM. Telephone assessment of cognition after transient ischemic attack and stroke: modified telephone interview of cognitive status and telephone Montreal Cognitive Assessment versus face-toface Montreal Cognitive Assessment and neuropsychological battery. Stroke. 2013;44(1):227-229. doi:10.1161/STROKEAHA.112.673384

37. Morley JE, Morris JC, Berg-Weger M, et al. Brain health: the importance of recognizing cognitive impairment: an IAGG consensus conference. J Am Med Dir Assoc. 2015;16(9):731-739. doi:10.10 16/j.jamda.2015.06.017 
38. Pohjasvaara T, Erkinjuntti T, Vataja R, Kaste M. Dementia three months after stroke. Baseline frequency and effect of different definitions of dementia in the Helsinki Stroke Aging Memory Study (SAM) cohort. Stroke. 1997;28(4):785-792. doi:10.1161/01.STR.28.4.785

39. Oveisgharan S, Hachinski V. Executive dysfunction is a strong stroke predictor. $J$ Neurol Sci. 2015;349(1-2):161-167. doi:10.1016/j. jns.2015.01.010

40. Wiberg B, Kilander L, Sundström J, Byberg L, Lind L. The relationship between executive dysfunction and post-stroke mortality: a population-based cohort study. BMJ Open. 2012;2(3):e458. doi:10.1136/bmjopen-2011-000458
41. Adams HJ, Davis PH, Leira EC, et al. Baseline NIH Stroke Scale score strongly predicts outcome after stroke: a report of the Trial of Org 10172 in Acute Stroke Treatment (TOAST). Neurology. 1999;53 (1):126-131. doi:10.1212/WNL.53.1.126

42. Pendlebury ST, Mariz J, Bull L, Mehta Z, Rothwell PM. MoCA, ACE-R, and MMSE versus the National Institute of Neurological Disorders and Stroke-Canadian Stroke Network vascular cognitive impairment harmonization standards neuropsychological battery after TIA and stroke. Stroke. 2012;43(2):464-469. doi:10.1161/STROKE AHA.111.633586

\section{Publish your work in this journal}

Clinical Interventions in Aging is an international, peer-reviewed journal focusing on evidence-based reports on the value or lack thereof of treatments intended to prevent or delay the onset of maladaptive correlates of aging in human beings. This journal is indexed on PubMed Central, MedLine, CAS, Scopus and the Elsevier
Bibliographic databases. The manuscript management system is completely online and includes a very quick and fair peer-review system, which is all easy to use. Visit http://www.dovepress.com testimonials.php to read real quotes from published authors. 